\title{
Trichuris suis ova in relapsing-remitting multiple sclerosis and clinically isolated syndrome (TRIOMS): study protocol for a randomized controlled trial
}

Berit Rosche ${ }^{1,2^{*}}$, Klaus-Dieter Wernecke ${ }^{3}$, Stephanie Ohlraun ${ }^{4}$, Jan-Markus Dörrr ${ }^{2,4}$ and Friedemann Paul ${ }^{2,4,5}$

\begin{abstract}
Background: Trichuris suis ova is a probiotic treatment based on the hygiene hypothesis. It has been demonstrated as safe and effective in autoimmune inflammatory bowel diseases and clinical trials indicate that helminth infections also have an immunomodulatory effect in multiple sclerosis.

We hypothesize that administering 2,500 Trichuris suis ova eggs orally every two weeks for 12 months is - due to its immunomodulatory and anti-inflammatory effect - significantly more effective than oral placebo in preventing new T2 and Gd+ lesions, as quantified by cerebral MRI and clinical examination, in relapsing-remitting multiple sclerosis and clinically isolated syndrome.
\end{abstract}

Methods/Design: Fifty patients with relapsing-remitting multiple sclerosis or clinically isolated syndrome with clinical activity, not undergoing any standard therapies, will be randomized 1:1 to Trichuris suis ova 2,500 eggs every two weeks or matching placebo. The safety, tolerability and effect on disease activity and in vivo mechanisms of action of Trichuris suis ova in MS will be assessed by neurological, laboratory and immunological exams and magnetic resonance imaging throughout the 12-month treatment period and over a follow-up period of 6 months. Various immunological analyses will be used to assess the overall patient immune response prior to and at varying time points following treatment with Trichuris suis ova.

Discussion: We anticipate that Trichuris suis ova will be well tolerated and more effective than the placebo in preventing new T2 and Gd+ lesions, as quantified by MRI. We also expect the Th1/Th17 proinflammatory response to shift towards the more anti-inflammatory Th2 response. This study has important clinical implications and will involve extensive research on the immunology of helminth therapy.

Trial registration: ClinicalTrials.gov: NCT01413243

Keywords: Multiple sclerosis, Trichuris suis ova, Clinical trial, Intervention, Immmunomodulation, Hygiene hypothesis

\section{Background}

Multiple sclerosis (MS) is a chronic inflammatory disease of the central nervous system (CNS), whose incidence has increased over recent decades [1] and which features a specific geographical distribution with higher incidence rates in developed Western nations [2,3].

\footnotetext{
* Correspondence: berit.rosche@charite.de

'Department of Neurology and Experimental Neurology, Charité

Universitätsmedizin Berlin, Charitéplatz 1, 10117 Berlin, Germany

${ }^{2}$ Clinical and Experimental Multiple Sclerosis Research Center, Charité -

Universitätsmedizin Berlin, Berlin, Germany

Full list of author information is available at the end of the article
}

Although the etiology remains unclear, MS is widely assumed to be an autoimmune disease, in which both genetic susceptibility and environmental factors play a role [4]. In 1966, Leibowitz et al. first suggested that MS might be associated with high sanitation standards during childhood [5], and subsequent epidemiological surveys have confirmed high MS prevalence in regions where such standards are the norm [6]. Beneficial immunomodulation by helminths in humans has been demonstrated in an observational study of relapsing-remitting (RR) MS patients with asymptomatic, community-acquired 
gastrointestinal infections [7]. Compared with uninfected MS patients, parasite-infected patients showed a significantly lower number of exacerbations, stable disability scores, and fewer new T2 and gadolinium enhancing $(\mathrm{Gd}+)$ lesions in brain magnetic resonance imaging (MRI). Consequently, new treatment paradigms based on the hygiene hypothesis were developed for autoimmune diseases, including the administering of non-pathogenic live microorganisms. The first clinical trials using Trichuris suis ova (TSO), a pig whipworm that is non-pathogenic in humans, in autoimmune diseases, such as inflammatory bowel disease, showed good tolerability and a striking suppressive effect on the autoimmune response [8]. Recently, Fleming et al. completed a phase I controlled trial that showed significant reduction of disease activity in RRMS patients under treatment with TSO [9]. We have also analyzed the effects of TSO in four patients with secondary progressive MS and observed a slight down-regulation of the Th1-associated cytokine pattern, especially interleukin (IL)-2, with a temporary increase of Th2-associated cytokines, such as IL-4. Furthermore, mild eosinophilia and changes in CD4+ and CD8+ T cells and natural killer (NK) CD56 bright cell numbers were evident [10].

A number of different hypotheses currently seek to explain how helminths influence autoimmune disorders. An increase in IL-10 and transforming growth factor- $\beta$ and a decrease in IL-12 and interferon- $\gamma$-secreting cells have been shown in fresh peripheral blood mononuclear cells (PBMCs) of MS patients with a helminth infection [7]. The patients also had a higher number of CD4+CD25+FoxP3+ regulatory $\mathrm{T}$ cells [7] and IL-10-producing CD19+ B cells [11]. The B cells also produced higher levels of brainderived neurotrophic factor and nerve growth factor. In the MS animal model experimental autoimmune encephalomyelitis (EAE), a reduced incidence and delayed onset of disease after infection with Schistosoma mansoni was seen, probably as a result of down-regulation of proinflammatory cytokines [12].

Overall, this research suggests that TSO is a promising therapeutic option in RRMS. We plan to investigate this further in a monocentric, prospective, randomized, placebo-controlled, double-blind phase II pilot study in patients with RRMS or clinically isolated syndrome (CIS) [13] using MRI and clinical assessment, and hypothesize that oral administration of 2,500 embryonated TSO every two weeks is superior to placebo administration.

\section{Methods/Design}

\section{Trial design}

TRIOMS is a monocentric, prospective, randomized, placebo-controlled, double-blind, phase II pilot study to be conducted at the Department of Neurology and the NeuroCure Clinical Research Center of the Charité -
Universitätsmedizin Berlin. We plan to begin recruitment in Summer 2012. Fifty patients with RRMS or CIS, who have not undergone immunomodulatory treatment for at least 3 months, will be randomized to either TSO 2,500 ova or placebo to be taken every two weeks for 12 months as an immunomodulatory monotherapy. The detailed study design is based on the revised Consolidated Standards of Reporting Trials Statement [14,15].

The study is approved by the local ethics committee and the German competent authority (Federal Institute for Drugs and Medical Devices). The trial is registered at Clinicaltrials.gov (NCT01413243) and will be conducted in accordance with the Declaration of Helsinki in its currently applicable version, the guidelines of the International Conference on Harmonization of Good Clinical Practice (ICH-GCP), and the applicable German laws. All participants will be required to give written informed consent. The trial will be monitored according to ICH-GCP.

\section{Participants}

The inclusion criteria for participation in the TRIOMS trial comprise the diagnosis of either definite RRMS according to the revised $2005 \mathrm{McDonald}$ criteria [16] or CIS, an age of 18 to 65 years, a score of $\leq 4.0$ on the Expanded Disability Status Scale (EDSS) [17], and disease activity on brain MRI (Table 1).

The main exclusion criteria include any disease course other than RRMS or CIS, any disease other than MS that may better explain the symptoms and signs, any immunomodulatory or immunosuppressive treatment, such as interferon- $\beta$, mitoxantrone, glatiramer acetate, natalizumab, within the preceding three months. Additionally, the participants must not have any significant uncontrolled disease, such as neoplasia or cardiovascular, renal, hepatic, endocrine or gastrointestinal disease or any other significant disease that may preclude the patient from participating in the study (Table 1).

\section{Randomization and interventions}

The eligibility of patients will be determined at the screening visit. At the baseline visit, patients who qualified for participation in the study will be stratified according to gender and disease course (RRMS/CIS) and randomized 1:1 into the two intervention groups. One group will receive TSO 2,500 eggs orally every 14 days, the other a placebo suspension orally every 14 days. Visits will be carried out after 3, 6, 9 and 12 months (Table 2). To ensure reliable monitoring of tolerability, telephone check-ups are planned after every dispensation if a patient does not visit the study center, because prior studies have shown frequent gastrointestinal adverse events or symptoms after therapy with TSO [18]. Optional follow-up visits will be offered at 15 and 


\section{Table 1 Eligibility criteria}

\begin{tabular}{ll}
\hline $\begin{array}{l}\text { Key inclusion } \\
\text { criteria }\end{array}$ & $\begin{array}{l}\text { Diagnosis of RRMS in accordance to the revised } \\
\text { McDonald criteria (2005) or CIS }\end{array}$
\end{tabular}

Aged 18 to 65 years, inclusive

Expanded Disability Status Scale (EDSS) at screening score $\leq 4.0$

Ability to provide written informed consent

Disease activity on brain defined by:

(A) $\geq 1$ gadolinium enhancing lesion on screening MRI or another MRI performed in the 12 months prior to screening OR

(B) $\geq 1$ new T2 lesion on screening MRI or on another MRI performed in the 12 months prior to screening in comparison to an earlier MRI performed in the previous 36 months OR

(C) $\geq 1$ enlarging T2 lesion on screening MRI or on another MRI performed in the 12 months before screening in comparison to an earlier MRI performed in the previous 36 months

Adequate birth control by a contraception method with a PEARL-index $<1$ in women of childbearing potential

Contraindication or intolerance for established standard immunomodulatory treatments with interferon- $\beta$ or glatiramer acetate

Information about established standard immunomodulatory treatments for MS by an independent neurologist and explicit decision of the patient against these treatment

Stable neurological state at study inclusion without any signs of a relapse and without steriod therapy in the last 30 days

Key exclusion Any disease other than MS that may better explain criteria the symptoms and signs

Any other immunomodulatory or immunosuppressive treatment, for example, interferon- $\beta$, mitoxantrone, glatiramer acetate, natalizumab within the preceding three months

Any significant uncontrolled disease, such as neoplasia, relevant liver disorder, active hepatitis B or $C$, and reduced liver function

Relevant laboratory findings:

- aspartate aminotransferase/alanine aminotransferase (ASAT/ALAT) $>3$ times of reference value

- bilirubine $>1.5 \mathrm{mg} / \mathrm{dl}$

- hemoglobin $<8.5 \mathrm{~g} / \mathrm{dl}$

- white blood count $<2.5 / \mathrm{nl}$

- thrombocytes $<125 / \mathrm{nl}$

- creatinine-clearance according to Cockroft-Gault -formula $<110 \mathrm{ml} / \mathrm{min}$ (male) and $<95 \mathrm{ml} / \mathrm{min}$ (female)

Any severe medical conditions or additional autoimmune disease which requires a immunosuppressive or immunomodulatory treatment

Any psychiatric or other condition that impedes the ability to provide informed consent or patient's compliance
Table 1 Eligibility criteria (Continued)

Refusal of transmission of personal data
Inability to complete an MRI scan
Relapse within 30 days of trial entry
Known allergies against components of TSO or
placebo
Participation in any interventional clinical trial in the
last three months or during the participant in
TRIOMS
Pregnancy or breast feeding
Concomitant medication with antihelminthic therapy
History of small intestinal resection

18 months after randomization. If necessary, additional unscheduled visits can be performed at any time.

\section{Outcome parameters}

The primary endpoint is the cumulative number of new hyperintense lesions identified on T2-weighted brain MRI during the treatment period of 12 months. Additional secondary MRI endpoints comprise the volume of T2 hyperintense lesions and number and volume of contrast-enhancing T1 hypointense lesions, the proportion of patients without any new $\mathrm{T} 1$ hypointense and $\mathrm{T} 2$ hyperintense lesions, brain atrophy as determined by brain parenchymal fraction and normalized brain volume 18 [19], and changes in brain metabolism (NAA/Cr-quotient) as determined by MR spectroscopy [20,21]. All MRI investigations will be performed using a 3 Tesla MRI scanner (Siemens Healthcare, Erlangen, Germany. Other secondary clinical endpoints include the annualized relapse rate and the proportion of relapse-free patients. A relapse is defined as de novo development, aggravation or re-occurrence of a preexisting neurological abnormality compatible with MS, which lasts a minimum of 24 hours, is separated by at least 30 days from a preceding clinical event and does not occur in the context of fever or infection. Further secondary endpoints are the disease progression as determined by EDSS (evaluated by an independent neurologist) [17], Multiple Sclerosis Functional Composite (MSFC) [22], depression as determined by the Beck Depression Inventory (BDI) [23] and fatigue as determined by the Fatigue Severity Scale (FSS) [24]. Assessment of all clinical and MRI endpoints will be performed by experienced evaluators blinded to both the clinical data and treatment allocation. Peripheral venous blood (serum, heparinized blood for flow cytometry and PBMC isolation) will be sampled prior to and during the intervention to assess the effect on cellular and soluble components of the immune system. In detail, we plan to 
Table 2 Time schedule of regular study visits

\begin{tabular}{|c|c|c|c|c|c|c|c|c|c|}
\hline & Screening & $\begin{array}{l}\text { Random } \\
\text { (month 0) }\end{array}$ & $\begin{array}{c}\text { Tel.V1 -6 } \\
\text { (week 0, 2, 4, } \\
6,8,10,16,20 \\
28,32,40,44) \\
\pm 3 \text { days }\end{array}$ & $\begin{array}{c}\text { V1 } \\
\text { (month } 3) \\
\pm 10 \text { days }\end{array}$ & $\begin{array}{c}\text { V2 } \\
\text { (month 6) } \\
\pm 10 \text { days }\end{array}$ & $\begin{array}{c}\text { V3 } \\
\text { (month 9) } \\
\pm 10 \text { days }\end{array}$ & $\begin{array}{c}\text { V4 } \\
\text { (month 12) } \\
\pm 10 \text { days }\end{array}$ & $\begin{array}{c}\text { V5 } \\
\text { (month 15) } \\
\pm 10 \text { days } \\
\text { (optional) }\end{array}$ & $\begin{array}{c}\text { V6 } \\
\text { (month 18) } \\
\pm 10 \text { days } \\
\text { (optional) }\end{array}$ \\
\hline Informed consent & $x$ & & & & & & & & \\
\hline In/exclusion criteria & $x$ & $x$ & & & & & & & \\
\hline Randomization & & $x$ & & & & & & & \\
\hline Demographic data & $x$ & & & & & & & & \\
\hline Pregnancy test & $x$ & & & & & & & & \\
\hline $\begin{array}{l}\text { Confirmation of MS } \\
\text { diagnosis }\end{array}$ & $x$ & & & & & & & & \\
\hline Relapse history & $x$ & $x$ & & $x$ & $x$ & $x$ & $x$ & $x$ & $x$ \\
\hline Medical history & $x$ & $x$ & & $x$ & $x$ & $x$ & $x$ & $x$ & $x$ \\
\hline Physical examination & $x$ & & & $x$ & $x$ & $x$ & $x$ & & $x$ \\
\hline Vital signs & $x$ & $x$ & & $x$ & $x$ & $x$ & $x$ & & $x$ \\
\hline Weight & $x$ & & & & & & $x$ & & $x$ \\
\hline MSFC & $x$ & & & & & & $x$ & & $x$ \\
\hline EDSS & $x$ & & $x$ & $x$ & $x$ & $x$ & $x$ & & $x$ \\
\hline Safety lab & $x$ & & & $x$ & $x$ & $x$ & $x$ & & $x$ \\
\hline $\begin{array}{l}\text { Immunological } \\
\text { sampling }\end{array}$ & & $x$ & & $x$ & $x$ & $x$ & $x$ & & $x$ \\
\hline MRI & $x$ & & & & $x$ & $x$ & $x$ & optional & optional \\
\hline AE/SAE & & & & $x$ & $x$ & $x$ & $x$ & $x$ & $x$ \\
\hline $\begin{array}{l}\text { Questionnaires } \\
\text { (fatigue, depression) }\end{array}$ & $x$ & $x$ & & & $x$ & $x$ & $x$ & $x$ & $x$ \\
\hline Drug supply & & $x$ & $x$ & $x$ & $x$ & $x$ & & & \\
\hline Drug account & & & & & $x$ & & $x$ & & \\
\hline
\end{tabular}

monitor the Th1/Th2/Th17 balance by concanavalin A stimulation of fresh heparinized blood and determination of cytokines using the cytokine bead array. In fresh heparinized blood, we will measure the frequency of B cells, CD4+ and CD8+ T cell subpopulations, innate immune cells and quantify the activation and homing markers on these cells by multicolor flow cytometry. We will also assess the number of IL-10-producing B cells by stimulation of fresh isolated PBMCs with $0.1 \mathrm{mg} / \mathrm{ml}$ CpG ODN2006 for $72 \mathrm{hr}$ and will add phorbol myristate acetate and ionomycin (PMA+iono) for the last $6 \mathrm{hr}$ and brefeldin A for the last $4 \mathrm{hr}$ in order to block cytokine excretion. Detection of IL-10 producing cells is planned as intracellular staining of IL-10 on CD 19 expressing cells by multicolor flow cytometry. Alternatively, IL-10 we will be measured in the culture supernatants by ELISA with aliquots of supernatant taken from cell cultures before the addition of brefeldin A and PMA+iono to the cultures.

\section{Sample size}

Because the present data on the effect of TSO on the development of new T2 hyperintense lesions on brain
MRI, which is the primary endpoint of the TRIOMS study, are insufficient for an exact statistical sample size calculation, we have designed the investigation as a pilot study with an a priori determined sample size of 25 patients per intervention arm, giving a total of 50 patients. The results of this exploratory analysis could serve as the basis for a further prospective randomized study with a statistically justified sample size.

\section{Blinding}

Both the patients and all study personnel will remain blinded throughout the complete treatment period of 12 months. Treatment allocations will only be disclosed after the final database lock or in case of emergency. The active study therapy and placebo bottles will be supplied by OVAMED GmbH and labeling of bottles and randomization of patients will be performed in the central pharmacy of the Charité - Universitätsmedizin Berlin. The patients will mainly be attended to by a "treating physician", responsible for evaluating the inclusion or exclusion criteria, adverse events, relapses, side effects and so on. All neurological examinations will be 
performed by an independent "evaluating physician". To separate the physicians' function in a threating and an evaluating position in order to prevent unblinding is necessary because patients under therapy with TSO commonly report gastrointestinal side effects that may point the physician to the verum therapy. Similarly, evaluation of all other paraclinical parameters, including MRI, will be conducted by independent examiners. Any patientrelated crosstalk between treating and evaluating physicians or examiners will be prohibited unless required for safety reasons. A simple unblinding procedure has been designed, which allows for rapid unblinding of a patient in case of a medical necessity. If the patient is unblinded, he or she will be automatically excluded from the study.

\section{Statistical methods}

Endpoints will be evaluated by both intention-to-treatand per-protocol-analyses. Statistical tests and presentation will be appropriate to the scaling and distribution of the respective variables. Because TRIOMS is planned as a pilot study, there is a statistical analysis of endpoints, but $P$-values will be considered exploratively without confirming generalization of hypotheses about the study treatments. Due to the restricted sample size of the pilot study, sufficiently powerful statistical analysis is not possible. The primary end point will be analyzed using Fisher's exact test. Secondary endpoints will be analyzed non-parametrically using Mann-Whitney-U-test, due to the small sample size and possible deviations from normality. Frequencies will be compared with the Fisher's exact test.Because of the exploratory character of the analysis, alpha-adjusting will not be performed. The test level for statistical significance of differences between both treatment arms is defined as $P=0.05$ (two-sided) for all tests. For statistical analyses use of the following software is planned: SAS, version 9.4 (SAS Institute Inc. Cary, North Carolina, US); SPSS, version 20 (IBM, Armonk, New York, US) and StatXact 6 from CYTEL (Cytel Inc., Cambridge, Massachusetts, US).

\section{Discussion}

Approved medications for MS, such as beta interferons or glatiramer acetate have limited therapeutic efficacy or, in the case of natalizumab, mitoxantrone or fingolimod, may be associated with severe side effects. The ideal drug for patients with MS should be highly effective with few and clinically insignificant side effects and should be administered orally. Existing epidemiological and preclinical data suggest that helminth infections are protective in autoimmune diseases, such as MS, and the first clinical trials with oral administration of pig whipworm Trichuris suis eggs in MS revealed good tolerance and surprising clinical effects $[9,10]$. Mechanisms of how helminth infections provide protection in autoimmune diseases are manifold and comprise immunosuppression attributed to an increased activity of regulatory $\mathrm{T}$ cells (Treg cells), alternatively activated macrophages and, more recently, IL-10 producing regulatory B cells. Further, helminth infections induce the production of Th2 related cytokines, such as IL-4, IL-5, IL-9 and IL-13, with anti-inflammatory qualities [25]. In addition, competitive mechanisms for antigen processing, antigen binding and for essential cytokines may exist between anti-infectious and autoimmune responses [26].

Here, we present a study design that has the potential to substantially contribute to the evaluation of the efficacy and tolerance of TSO in MS patients. The randomized, controlled, double-blind study design and the implementation of independent evaluation of outcome parameters fulfill the current criteria for a high quality clinical phase II trial in MS $[14,15]$. A limited sample size was chosen to reduce the number of patients on placebo and on a possibly ineffective therapy. On the other hand, this limited sample size may not be sufficient to show a statistically significant superiority of TSO compared with placebo. The placebo design was chosen to evaluate the sole effect of TSO on MS activity and to minimize the risk of uncontrolled immunosuppression that could be relevant in a treatment with TSO and other immunosuppressive or immunomodulatory therapies. Fleming et al. showed a reduction of $\mathrm{Gd}+$ lesions in MRI under therapy with TSO in MS patients after three months of therapy and an increase of lesions in the post-treatment period, suggesting that a permanent therapy is desirable for treatment of a chronic disease like MS [9]. Therefore, we have chosen a relatively long therapy duration of 12 months to confirm on the one hand the clinical effects of TSO on MS activity and to show on the other hand that this medication fulfills safety aspects for long time use.

In conclusion, TSO has the potential as an effective, safe and orally available treatment option in MS and the TRIOMS trial may help to establish the first probiotic therapy in MS that is based on the hygiene hypothesis.

\section{Trial status}

Start of recruitment September 2012.

\section{Abbreviations}

AE: Adverse event; ALAT: Alanine aminotransferase; ASAT: Aspartate aminotransferase; BDI: Beck Depression Inventory; CIS: Clinically isolated syndrome; CNS: Central nervous system; EAE: Experimental autoimmune encephalomyelitis; EDSS: Expanded disability status scale; FSS: Fatigue Severity Scale; ICH-GCP: International Conference on Harmonization of Good Clinical Practice; IL: Interleukin; MS: Multiple sclerosis; MSFC: Multiple sclerosis functional composite; MRI: Magnetic resonance imaging; NK: Natural killer; PBMCs: Peripheral blood mononuclear cells; RR: Relapsing-remitting; SAE: Serious adverse event; TRIOMS: Trichuris suis ova in relapsing-remitting multiple sclerosis and clinically isolated syndrome; TSO: Trichuris suis ova; V: Visit. 


\section{Competing interests}

BR participated in meetings sponsored by and received lecture honoraria from Biogen Idec, Bayer, Merck Sorono and Teva Pharmaceuticals, and receives research support from Bayer Schering Pharma. FP received travel support and speaker honoraria by Bayer Healthcare, Novartis, Teva, Biogen Idec, Sanofi Aventis and Merck Serono. JD received travel support, speaker honoraria and research support by Novartis and Bayer Vital. The authors declare that they have no competing interests.

\section{Authors' contributions}

BR designed the study, drafted the study protocol and drafted the manuscript. SO was in charge of all regulatory affairs and critically revised the study protocol and manuscript. KDW is the biometrician of the study and critically revised the study protocol and manuscript. FP designed the study and also critically revised the study protocol and manuscript. JD critically revised the study protocol and manuscript. All authors have given final approval of the version to be published.

\section{Acknowledgements}

\section{Funding}

Trichuris suis ova and placebo for the TRIOMS trial is provided by OVAMED $\mathrm{GmbH}$, Barsbüttel.

The TRIOMS trial is supported by the German Research Foundation (DFG Exc 257).

\section{Author details}

'Department of Neurology and Experimental Neurology, Charité Universitätsmedizin Berlin, Charitéplatz 1, 10117 Berlin, Germany. ${ }^{2}$ Clinical and Experimental Multiple Sclerosis Research Center, Charité - Universitätsmedizin Berlin, Berlin, Germany. ${ }^{3}$ SOSTANA GmbH and Charité, Universitätsmedizin Berlin, Berlin, Germany. ${ }^{4}$ NeuroCure Clinical Research Center, Charité Universitätsmedizin Berlin, Berlin, Germany. ${ }^{5}$ Experimental and Clinical Research Center, Max Delbrueck Center for Molecular Medicine and Charité Universitätsmedizin Berlin, Berlin, Germany.

Received: 12 September 2012 Accepted: 8 April 2013

Published: 25 April 2013

\section{References}

1. Hirst C, Ingram G, Pickersgill T, Swingler R, Compston DAS, Robertson NP: Increasing prevalence and incidence of multiple sclerosis in South East Wales. J Neurol Neurosurg Psychiatry 2009, 80:386-391.

2. Hernan MA, Olek MJ, Ascherio A: Geographic variation of MS incidence in two prospective studies of US women. Neurology 1999, 53:1711-1718.

3. Kurtzke JF, Beebe GW, Norman JE Jr: Epidemiology of multiple sclerosis in US veterans: III. Migration and the risk of MS. Neurology 1985, 35:672-678.

4. Compston A, Coles A: Multiple sclerosis. Lancet 2008, 372:1502-1517.

5. Leibowitz U, Antonovsky A, Medalie JM, Smith HA, Halpern L, Alter M: Epidemiological study of multiple sclerosis in Israel. II. Multiple sclerosis and level of sanitation. J Neurol Neurosurg Psychiatry 1966, 29:60-68.

6. Fleming JO, Cook TD: Multiple sclerosis and the hygiene hypothesis. Neurology 2006, 67:2085-2086.

7. Correale J, Farez M: Association between parasite infection and immune responses in multiple sclerosis. Ann Neurol 2007, 61:97-108.

8. Summers RW, Elliott DE, Qadir K, Urban JF, Thompson R, Weinstock JV: Trichuris suis seems to be safe and possibly effective in the treatment of inflammatory bowel disease. Am J Gastroenterol 2003, 98:2034-2041.

9. Fleming JO, Isaak A, Lee JE, Luzzio CC, Carrithers MD, Cook TD, Field AS, Boland J, Fabry Z: Probiotic helminth administration in relapsingremitting multiple sclerosis: a phase 1 study. Mult Scler 2011, 17:743-754.

10. Benzel F, Erdur H, Kohler S, Frentsch M, Thiel A, Harms L, Wandinger KP, Rosche B: Immune monitoring of Trichuris suis egg therapy in multiple sclerosis patients. J Helminthol 2012, 86:339-347.

11. Correale J, Farez M, Razzitte G: Helminth infections associated with multiple sclerosis induce regulatory B cells. Ann Neurol 2008, 64:187-199.

12. La Flamme AC, Ruddenklau K, Backstrom BT: Schistosomiasis decreases central nervous system inflammation and alters the progression of experimental autoimmune encephalomyelitis. Infect Immun 2003, 71:4996-5004

13. McDonald WI, Compston A, Edan G, Goodkin D, Hartung HP, Lublin FD, McFarland HF, Paty DW, Polman CH, Reingold SC, Sandberg-Wollheim M,
Sibley W, Thompson A, van den Noort S, Weinshenker BY, Wolinsky JS: Recommended diagnostic criteria for multiple sclerosis: guidelines from the International Panel on the Diagnosis of Multiple Sclerosis. Ann Neurol 2001, 50:121-127.

14. Moher D, Schulz KF, Altman DG: The CONSORT statement: revised recommendations for improving the quality of reports of parallel group randomized trials. BMC Med Res Methodol 2001, 1:2.

15. Schulz KF, Altman DG, Moher D: CONSORT 2010 Statement: updated guidelines for reporting parallel group randomised trials. Trials 2010, 11:32.

16. Polman CH, Reingold SC, Edan G, Filippi M, Hartung HP, Kappos L, Lublin FD, Metz LM, McFarland HF, O'Connor PW, Sandberg-Wollheim M, Thompson AJ, Weinshenker BG, Wolinsky JS: Diagnostic criteria for multiple sclerosis: 2005 Revisions to the "McDonald Criteria". Ann Neurol 2005, 58:840-846.

17. Kurtzke JF: Rating neurologic impairment in multiple sclerosis: an expanded disability status scale (EDSS). Neurology 1983, 33:1444-1452

18. Bager P, Kapel C, Roepstorff A, Thamsborg S, Arnved J, Rønborg S, Kristensen B, Poulsen LK, Wohlfahrt J, Melbye M: Symptoms after ingestion of pig whipworm Trichuris suis eggs in a randomized placebo-controlled double-blind clinical trial. PLoS One 2011, 6:e22346.

19. Zimmermann H, Freing A, Kaufhold F, Gaede G, Bohn E, Bock M, Oberwahrenbrock T, Young KL, Dörr J, Wuerfel JT, Schippling S, Paul F, Brandt AU: Optic neuritis interferes with optical coherence tomography and magnetic resonance imaging correlations. Mult Scler 2013, 19:443-450.

20. Dörr J, Wernecke KD, Bock M, Gaede G, Wuerfel JT, Pfueller CF, BellmannStrobl J, Freing A, Brandt AU, Friedemann P: Association of retinal and macular damage with brain atrophy in multiple sclerosis. PLoS One 2011, 6:e18132.

21. Pfueller CF, Brandt AU, Schubert F, Bock M, Walaszek B, Waiczies $H$, Schwenteck T, Dörr J, Bellmann-Strobl J, Mohr C, Weinges-Evers N, Ittermann B, Wuerfel JT, Paul F: Metabolic changes in the visual cortex are linked to retinal nerve fiber layer thinning in multiple sclerosis. PLoS One 2011, 6:e18019.

22. Cutter GR, Baier ML, Rudick RA, Cookfair DL, Fischer JS, Petkau J, Syndulko K, Weinshenker BG, Antel JP, Confavreux C, Ellison GW, Lublin F, Miller AE, Rao SM, Reingold S, Thompson A, Willoughby E: Development of a multiple sclerosis functional composite as a clinical trial outcome measure. Brain 1999, 122:871-882.

23. Beck AT, Ward CH, Mendelson M, Mock J, Erbaugh J: An inventory for measuring depression. Arch Gen Psychiatry 1961, 4:561-571.

24. Krupp LB, LaRocca NG, Muir-Nash J, Steinberg AD: The fatigue severity scale. Application to patients with multiple sclerosis and systemic lupus erythematosus. Arch Neurol 1989, 46:1121-1123.

25. Gaze S, McSorley HJ, Daveson J, Jones D, Bethony JM, Oliveira LM, Speare R, McCarthy JS, Engwerda CR, Croese J, Loukas A: Characterising the mucosal and systemic immune responses to experimental human hookworm infection. PLoS Pathog 2012, 8:e1002520.

26. Correale J, Farez MF: The impact of parasite infections on the course of multiple sclerosis. J Neuroimmunol 2011, 233:6-11.

doi:10.1186/1745-6215-14-112

Cite this article as: Rosche et al:: Trichuris suis ova in relapsing-remitting multiple sclerosis and clinically isolated syndrome (TRIOMS): study protocol for a randomized controlled trial. Trials 2013 14:112. 